\title{
Dense coding for continuous variables
}

\author{
Samuel L. Braunstein \\ Informatics, University of Wales, Bangor LL57 1UT, United Kingdom
}

H. J. Kimble

Norman Bridge Laboratory of Physics 12-33, California Institute of Technology, Pasadena, California 91125

(Received 3 May 1999; published 3 March 2000)

\begin{abstract}
A scheme to achieve dense quantum coding for the quadrature amplitudes of the electromagnetic field is presented. The protocol utilizes shared entanglement provided by nondegenerate parametric down-conversion in the limit of large gain to attain high efficiency. For a constraint in the mean number of photons $\bar{n}$ associated with modulation in the signal channel, the channel capacity for dense coding is found to be $\ln \left(1+\bar{n}+\bar{n}^{2}\right)$, which always beats coherent-state communication and surpasses squeezed-state communication for $\bar{n}>1$. For $\bar{n}$ $\gg 1$, the dense coding capacity approaches twice that of either scheme.

PACS number(s): 03.67.Hk, 42.50.Dv
\end{abstract}

An important component of contemporary quantum information theory is the investigation of the classical information capacities of noisy quantum communication channels. Here, classical information is encoded by the choice of one particular quantum state from among a predefined ensemble of quantum states by the sender Alice for transmission over a quantum channel to the receiver Bob. If Alice and Bob are allowed to communicate only via a one-way exchange along such a noisy quantum channel, then the optimal amount of classical information that can be reliably transmitted over the channel has recently been established $[1,2]$.

Stated more explicitly, if a classical signal $\alpha$ taken from the ensemble $P_{\alpha}$ is to be transmitted as a quantum state $\hat{\rho}_{\alpha}$, then Holevo's bound for a bosonic quantum channel says that the mutual information $H(A: B)$ between the sender $A$ (Alice) and receiver $B(\mathrm{Bob})$ is bounded by [1]

$$
H(A: B) \leqslant S(\hat{\rho})-\int d^{2} \alpha P_{\alpha} S\left(\hat{\rho}_{\alpha}\right) \leqslant S(\hat{\rho}),
$$

where $S(\hat{\rho})$ is the von Neumann entropy associated with the density operator $\hat{\rho}=\int d^{2} \alpha P_{\alpha} \hat{\rho}_{\alpha}$ for the mean channel state.

By contrast, if Alice and Bob share a quantum resource in the form if an ensemble of entangled states, then quantum mechanics enables protocols for communication that can circumvent the aforementioned bound on channel capacity. For example, as shown originally by Bennett and Wiesner [3], Alice and Bob can beat the Holevo limit by exploiting their shared entanglement to achieve dense quantum coding. Here, the signal is encoded at Alice's sending station and transmitted via one component of a pair of entangled quantum states, with then the second component of the entangled pair exploited for decoding the signal at Bob's receiving station. In this scheme, the cost of distributing the entangled states to Alice and Bob is not figured into the accounting of constraints on the quantum channel (e.g., the mean energy). Such neglect of the distribution cost of entanglement is sensible in some situations, as for example, if the entanglement were to be sent during off-peak times when the communication channel is otherwise under utilized, or if it had been conveyed by other means to Alice and Bob in advance (e.g., via a pair of quantum $C D s$ with stored, entangled quantum states). Note that in general, no signal modulation is applied to the second (i.e., Bob's) component of the entangled state, so that it carries no information by itself.

Although quantum dense coding has most often been discussed within the setting of discrete quantum variables (e.g., qubits) $[3,4]$, in this paper we show that highly efficient dense coding is possible for continuous quantum variables. As in our prior work on quantum teleportation [5-7], our scheme for achieving quantum dense coding exploits squeezed-state entanglement, and therefore should allow unconditional signal transmission with high efficiency, in contrast to the conditional transmission with extremely low efficiency achieved in Ref. [4]. More specifically, for signal states $\alpha$ associated with the complex amplitude of the electromagnetic field, the channel capacity for dense coding is found to be $\ln \left(1+\bar{n}+\bar{n}^{2}\right)$, where $\bar{n}$ is the mean photon number for modulation in the signal channel. The channel capacity for dense coding in our scheme thus always beats coherent-state communication and surpasses squeezed-state communication for $\bar{n}>1$. For $\bar{n} \gg 1$, the dense coding capacity approaches twice that of either scheme.

As illustrated in Fig. 1, the relevant continuous variables for our protocol are the quadrature amplitudes $(\hat{x}, \hat{p})$ of the electromagnetic field, with the classical signal $\alpha=\langle\hat{x}\rangle$ $+i\langle\hat{p}\rangle$ then associated with the quantum state $\hat{\rho}_{\alpha}$ drawn from the phase space for a single mode of the field. The entangled resource shared by Alice and Bob is a pair of EPR beams with quantum correlations between canonically conjugate variables $(\hat{x}, \hat{p})_{(1,2)}$ as were first described by Einstein, Podolsky, and Rosen (EPR [8]), and which can be efficiently generated via the nonlinear optical process of parametric down conversion, resulting in a highly squeezed two-mode state of the electromagnetic field $[9,10]$. In the ideal case, the correlations between quadrature-phase amplitudes for the two beams $(1,2)$ are such that

$$
\left\langle\left(\hat{x}_{1}-\hat{x}_{2}\right)^{2}\right\rangle \rightarrow 0, \quad\left\langle\left(\hat{p}_{1}+\hat{p}_{2}\right)^{2}\right\rangle \rightarrow 0,
$$




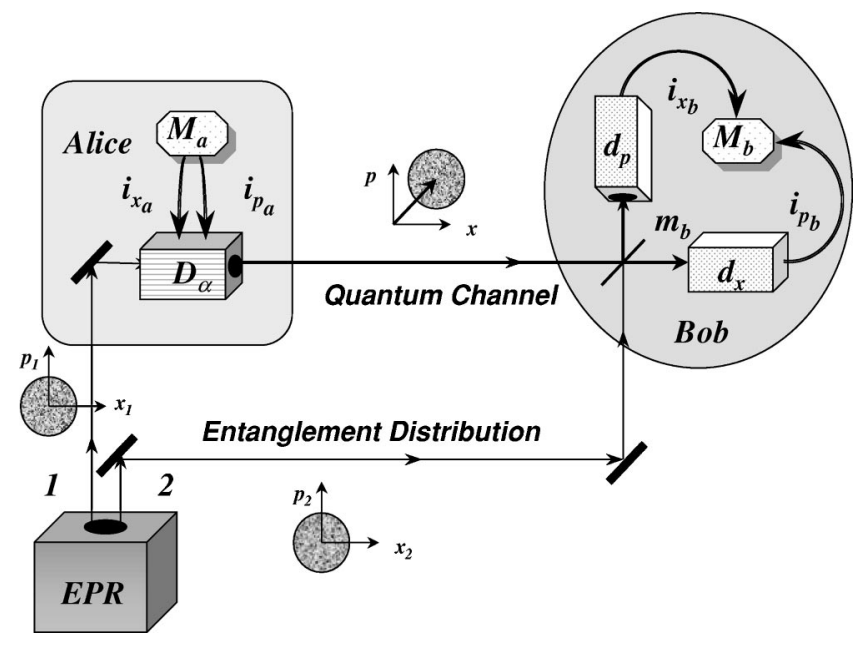

FIG. 1. Illustration of the scheme for achieving super-dense quantum coding for signal states over the complex amplitude $\alpha$ $=x+i p$ of the electromagnetic field. The quantum resource that enables dense coding is the EPR source that generates entangled beams $(1,2)$ shared by Alice and Bob.

albeit it with an concomitant divergence in the mean photon number $\bar{n}$ in each channel.

Component 1 of this entangled pair of beams is input to Alice's sending station, where the message $M_{a}^{\alpha}$ corresponding to the classical signal $\alpha_{\text {in }}$ is encoded as the quantum state $\hat{\rho}_{\alpha_{\text {in }}}$ by a simple phase-space offset by way of the displacement operator $\hat{D}\left(\alpha_{\text {in }}\right)$ applied to 1 [11]. The displacement $\hat{D}\left(\alpha_{\text {in }}\right)$ can be implemented in a straightforward fashion by amplitude and phase offsets generated by the (suitably normalized) classical currents $\left(i_{x_{a}}, i_{p_{a}}\right)$ as in Ref. [7]. The state corresponding to Alice's displacement of the EPR beam constitutes the quantum signal and is transmitted along the quantum channel shown in Fig. 1 to Bob's receiving station, (Fig. 2) where it is decoded with the aid of the second component 2 of the original EPR pair of beams and the homodyne detectors $\left(d_{x}, d_{p}\right)$. The resulting photocurrents $\left(i_{x_{b}}, i_{p_{b}}\right)$ suitably normalized to produce $\alpha_{\text {out }}=i_{x_{b}}+i i_{p_{b}}$ constitute the message $M_{b}^{\alpha}$ received by Bob. In the limit $\bar{n} \rightarrow \infty$, Eq. (2) ensures $\alpha_{\text {out }}=\alpha_{\text {in }}$, so that the classical message would be perfectly recovered. However, even for finite $\bar{n}$ as is relevant to a channel constrained in mean energy, the finite correlations implicit in the EPR beams enable quantum dense coding with enhanced channel capacity relative to either coherent state or squeezed state communication, as we now show.

Consider the specific case of EPR beams $(1,2)$ approximated by the two-mode squeezed state with Wigner function

$$
\begin{aligned}
W_{\mathrm{EPR}}\left(\alpha_{1}, \alpha_{2}\right)= & \frac{4}{\pi^{2}} \exp \left[-e^{-2 r}\left(\alpha_{1}-\alpha_{2}\right)_{R}^{2}-e^{2 r}\left(\alpha_{1}-\alpha_{2}\right)_{I}^{2}\right. \\
& \left.-e^{2 r}\left(\alpha_{1}+\alpha_{2}\right)_{R}^{2}-e^{-2 r}\left(\alpha_{1}+\alpha_{2}\right)_{I}^{2}\right],
\end{aligned}
$$

where the subscripts $R$ and $I$ refer to real and imaginary parts

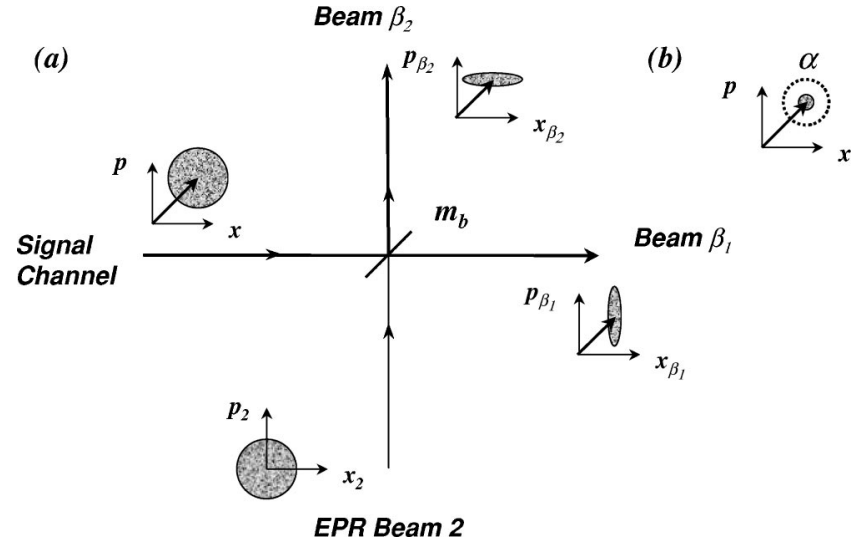

FIG. 2. Depiction of signal decoding at Bob's receiving station. (a) At Bob's $50-50$ beam splitter $m_{b}$, the displaced EPR beam 1 is combined with the component 2 to yield two independent squeezed beams, with the $\beta_{1,2}$ beams having fluctuations reduced below the vacuum-state limit along $\left(x_{\beta_{1}}, p_{\beta_{2}}\right)$. Homodyne detection at $\left(d_{x}, d_{p}\right)$ (Fig. 1) with LO phases set to measure $\left(x_{\beta_{1}}, p_{\beta_{2}}\right)$, respectively, then yields the complex signal amplitude $\alpha_{\text {out }}$ with variance set by the associated squeezed states. (b) The net effect of the dense coding protocol is the transmission and detection of states of complex amplitude $\alpha$ with an effective uncertainty below the vacuumstate limit (indicated by the dashed circle).

of the field amplitude $\alpha$, respectively (i.e., $\alpha_{R, I}=x, p$ ). Note that for $r \rightarrow \infty$, the field state becomes the ideal EPR state as described in Eq. (2), namely,

$$
W_{\mathrm{EPR}}\left(\alpha_{1}, \alpha_{2}\right) \rightarrow C \delta\left(\alpha_{1 R}+\alpha_{2 R}\right) \delta\left(\alpha_{1 I}-\alpha_{2 I}\right) .
$$

As shown in Fig. 1, signal modulation is performed only on mode 1, with mode 2 treated as an overall shared resource by Alice and Bob (and which could have been generated by Alice herself). The modulation scheme that we choose is simply to displace mode 1 by an amount $\alpha_{\text {in }}$. This leads to a displaced Wigner function given by $W_{\mathrm{EPR}}\left(\alpha_{1}\right.$ $-\alpha_{\text {in }}, \alpha_{2}$ ), corresponding to the field state that is sent via the quantum channel from Alice to Bob.

Upon receiving this transmitted state (consisting of the modulated mode 1), the final step in the dense-coding protocol is for Bob to combine it with the shared resource (mode 2 ) and retrieve the original classical signal $\alpha_{\text {in }}$ with as high a fidelity as possible. As indicated in Fig. 1, this demodulation can be performed with a simple 50-50 beam splitter that superposes the modes $(1,2)$ to yield output fields that are the sum and difference of the input fields and which we label as $\beta_{1}$ and $\beta_{2}$, respectively. The resulting state emerging from Bob's beam splitter has Wigner function

$$
W_{\text {sum } / \text { diff }}\left(\beta_{1}, \beta_{2}\right)=W_{\mathrm{EPR}}\left(\left(\beta_{1}+\beta_{2}\right) / \sqrt{2}-\alpha,\left(\beta_{1}-\beta_{2}\right) / \sqrt{2}\right) \text {. }
$$

The classical signal that we seek is retrieved by homodyne detection at detectors $\left(d_{x}, d_{p}\right)$, which measure the analogs of position and momentum for the sum and difference fields $\left(\beta_{1}, \beta_{2}\right)$. For ideal homodyne detection the resulting outcomes are distributed according to 


$$
P(\beta \mid \alpha)=\frac{2 e^{2 r}}{\pi} \exp \left(-2 e^{2 r}|\beta-\alpha / \sqrt{2}|^{2}\right),
$$

where $\beta=\beta_{1 R}+i \beta_{2 I}$ and represents a highly peaked distribution about the complex displacement $\alpha / \sqrt{2}$. For large squeezing parameter $r$ this allows us to extract the original signal $\alpha$ which we choose to be distributed as

$$
P_{\alpha}=\frac{1}{\pi \sigma^{2}} \exp \left(-|\alpha|^{2} / \sigma^{2}\right)
$$

Note that mode 1 of this displaced state has a mean number of photons given by

$$
\bar{n}=\sigma^{2}+\sinh ^{2} r .
$$

In order to compute the quantity of information that may be sent through this dense coding channel we note the unconditioned probability for the homodyne statistics is given by

$$
P(\beta)=\frac{2}{\pi\left(\sigma^{2}+e^{-2 r}\right)} \exp \left(\frac{-2|\beta|^{2}}{\sigma^{2}+e^{-2 r}}\right) .
$$

The mutual information describing the achievable information throughput of this dense coding channel is then given by

$$
\begin{aligned}
H^{\text {dense }}(A: B) & =\int d^{2} \beta d^{2} \alpha P(\beta \mid \alpha) P_{\alpha} \ln \left(\frac{P(\beta \mid \alpha)}{P(\beta)}\right) \\
& =\ln \left(1+\sigma^{2} e^{2 r}\right) .
\end{aligned}
$$

For a fixed $\bar{n}$ in Eq. (7) this information is optimized when $\bar{n}=e^{r} \sinh r$, i.e., when $\sigma^{2}=\sinh r \cosh r$ so yielding a dense coding capacity of

$$
C^{\text {dense }}=\ln \left(1+\bar{n}+\bar{n}^{2}\right),
$$

which for large squeezing $r$ becomes

$$
C^{\text {dense }} \sim 4 r .
$$

How efficient is this dense coding in comparison to single channel coding? Let us place a "common" constraint of having a fixed mean number of photons $\bar{n}$ which can be modulated. For a single bosonic channel Drummond and Caves [12] and Yuen and Ozawa [13] have used Holevo's result to show that the optimal channel capacity is just that given by photon counting from a maximum entropy ensemble of number states. In this case the channel capacity (the maximal mutual information) achieves the ensemble entropy, see Eq. (1), so

$$
C=S(\rho)=(1+\bar{n}) \ln (1+\bar{n})-\bar{n} \ln \bar{n} .
$$

Substituting $\bar{n}=e^{r} \sinh r$ into this we find

$$
C \sim 2 r,
$$

for large squeezing $r$. This is just one-half of the asymptotic dense coding mutual information, see Eq. (11). Thus asymptotically, at least, the dense coding scheme allows twice as much information to be encoded within a given state, although it has an extra expense (not included within the simple constraint $\bar{n}$ ) of requiring shared entanglement.

It is worth noting that this dense coding scheme does not always beat the optimal single channel capacity. Indeed, for small squeezing it is worse. The break-even squeezing required for dense coding to equal the capacity of the optimal single channel communication is

$$
r_{\text {break-even }} \simeq 0.7809,
$$

which corresponds to roughly $6.78 d B$ of two-mode squeezing or to $\bar{n} \simeq 1.884$. This break-even point takes into account the difficulty of making highly squeezed two-mode squeezed states. No similar difficulty has been factored into making ideal number states used in the benchmark scheme with which our dense coding scheme is compared.

A fairer comparison is against single-mode coherent state communication with heterodyne detection. Here the channel capacity is well known [14-16] for the mean photon number constraint to be

$$
C^{\mathrm{coh}}=\ln (1+\bar{n}),
$$

which is always beaten by the optimal dense coding scheme described by Eq. (10).

An improvement on coherent state communication is squeezed state communication with a single mode. The channel capacity of this channel has been calculated [16] to be

$$
C^{\mathrm{sq}}=\ln (1+2 \bar{n}),
$$

which is beaten by the dense coding scheme of Eq. (10) for $\bar{n}>1$, i.e., the break-even squeezing required is

$$
r_{\text {break-even }}^{\text {sq }} \simeq 0.5493,
$$

which corresponds to $4.77 d B$.

In summary, we have shown how to perform dense quantum coding for continuous quantum variables by utilizing squeezed state entanglement. For a constraint in the mean number of photons that may be modulated $\bar{n}$, the dense coding capacity is found to be $\ln \left(1+\bar{n}+\bar{n}^{2}\right)$. This scheme always beats single-mode coherent-state communication and surpasses single-mode squeezed-state communication for $\bar{n}$ $>1$. Note that in terms of actual implementation, our protocol should allow for high efficiency, unconditional transmission with encoded information sent every inverse bandwidth time. This situation is in contrast to implementations that employ weak parametric down conversion, where transmission is achieved conditionally and relatively rarely. In fact Mattle et al. [4] obtained rates of only 1 in $10^{7}$ per inverse bandwidth time [17]. By going to strong down conversion and using a characteristically different type of entanglement, our scheme should allow information to be sent with much higher efficiency and should simultaneously improve the 
ability to detect orthogonal Bell states. Indeed, these advantages enabled the first experimental realization of unconditional quantum teleportation within the past year [7]. Beyond the particular setting of quantum communication discussed here, this research is part of a larger program to explore the potential for quantum information processing with continuous quantum variables. Such investigations are quite timely in light of important recent progress concerning the prospects for diverse quantum algorithms with continuous variables, including universal quantum computation [18] and quantum error correction [19-21], with quantum teleportation being a prime example $[5,22,23]$. Although still in its earliest stages, theoretical protocols have been developed for realistic physical systems that should allow a variety of elementary processing operations for continuous quantum variables, including significantly quantum storage for EPR states. [24,25]

S.L.B. was supported in part by the UK Engineering and Physical Sciences Research Council and the Royal Academy of Engineering. The work of H.J.K. is supported by DARPA via the QUIC Institute which is administered by ARO, by the Office of Naval Research, and by the National Science Foundation.
[1] A.S. Holevo, IEEE Trans. Inf. Theory 44, 269 (1998).

[2] B. Schumacher and M.D. Westmoreland, Phys. Rev. A 56, 131 (1997).

[3] C.H. Bennett and S.J. Wiesner, Phys. Rev. Lett. 69, 2881 (1992).

[4] K. Mattle, H. Weinfurter, P.G. Kwiat, and A. Zeilinger, Phys. Rev. Lett. 76, 4656 (1996).

[5] S.L. Braunstein and H.J. Kimble, Phys. Rev. Lett. 80, 869 (1998).

[6] P. van Loock, S.L. Braunstein, and H.J. Kimble, LANL e-print quant-ph/9902030.

[7] A. Furusawa, J. Sørensen, S.L. Braunstein, C. Fuchs, H.J. Kimble, and E.S. Polzik, Science 282, 706 (1998).

[8] A. Einstein, B. Podolsky, and N. Rosen, Phys. Rev. 47, 777 (1935).

[9] M.D. Reid and P.D. Drummond, Phys. Rev. Lett. 60, 2731 (1988); M.D. Reid, Phys. Rev. A 40, 913 (1989).

[10] Z.Y. Ou, S.F. Pereira, H.J. Kimble, and K.C. Peng, Phys. Rev. Lett. 68, 3663 (1992); Appl. Phys. B: Photophys. Laser Chem. 55, 265 (1992).
[11] L. Mandel and E. Wolf, Optical Coherence and Quantum Optics (Cambridge, Cambridge University Press, Cambridge, England, 1995).

[12] C.M. Caves and P.D. Drummond, Rev. Mod. Phys. 66, 481 (1994).

[13] H.P. Yuen and M. Ozawa, Phys. Rev. Lett. 70, 363 (1993).

[14] J.P. Gordon, Proc. IRE 50, 1898 (1962).

[15] C.Y. She, IEEE Trans. Inf. Theory IT-14, 32 (1968).

[16] Y. Yamamoto and H.A. Haus, Rev. Mod. Phys. 58, 1001 (1986).

[17] H. Weinfurter (private communication).

[18] S. Lloyd and S.L. Braunstein, Phys. Rev. Lett. 82, 1784 (1999).

[19] S. Lloyd and J.J.-E. Slotine, Phys. Rev. Lett. 80, 4088 (1998).

[20] S.L. Braunstein, Phys. Rev. Lett. 80, 4084 (1998).

[21] S.L. Braunstein, Nature (London) 394, 47 (1998).

[22] C.H. Bennett et al., Phys. Rev. Lett. 70, 1895 (1993).

[23] L. Vaidman, Phys. Rev. A 49, 1473 (1994).

[24] A.S. Parkins and H.J. Kimble, e-print quant-ph/9904062.

[25] A.S. Parkins and H.J. Kimble, e-print quant-ph/9907049. 